\title{
An improved bat optimization algorithm of Sports Video
}

\author{
Qingmei Liu,a
}

\author{
${ }^{1}$ Gansu Normal University for Nationalities, Hezuo, gansu, 747000, China \\ asuonanhuadan@163.com
}

Keywords: Bat optimization algorithm; Maneuver flight; Sports Video; intelligent algorithms; Application

\begin{abstract}
In this paper, an improved bat optimization algorithm to solve sports video was proposed, which presented the concept of adjustment sequence to design the strategy of local searching, and added the maneuver flight in the global exchange of information. Experimental results indicate that, the proposed algorithm has more powerful search capability and more strong robustness in solving sports video.
\end{abstract}

\section{Introduction}

The bat algorithm (BA) is a random search optimization algorithm, it is proposed by Yang in $2010^{[1]}$. BA algorithm has the advantages of simple theoretical method, less setting parameters and easy implementation of encoding. Since BA algorithm was proposed, it has been applied to optimization problem. Such as, the algorithm was used to solve the multi-objective optimization problem by Yang in $2011^{[2]}$, the algorithm shows a good performance in the design of welding beam. In the same year, Tsaid proposed an improved BA algorithm for solving numerical optimization problems ${ }^{[3]}$. In 2011, K Khan proposed a fuzzy clustering BA algorithm for solving the problem of workplace screening, which was used to solve the problem of workplace screening, and extracted the relevant data from the workplace health risk index, and then clustered with BA algorithm $^{[4]}$. In the same year, A L Tamiru Successfully applied BA algorithm and fuzzy system to the energy model of gas turbine ${ }^{[5]}$. The main components of the gas turbine are discussed by using fuzzy system. BA algorithm is used to fuzzy clustering by Mishra S in $2012^{[6]}$. BA algorithm was applied to solve the problem of Brushless DC motor by Bora $\mathrm{T} \mathrm{C}^{[7]}$.

BA algorithm is still in the development stage, foreign scholars have successfully applied the BA algorithm to optimize the problem, but the domestic scholars study the BA algorithm is relatively small, in 2012 Li Zhiyong proposed An improved bat optimization algorithm, in the algorithm, the introduction of genetic variation into the BA algorithm, and the improved algorithm is successfully applied to 0-1 knapsack problem ${ }^{[8]}$. In 2013 , the global convergence of the algorithm for solving large scale optimization problems was proposed by Huang Guangqiu ${ }^{[9]}$. In $2014 \mathrm{He}$ Xingshi proposed a simulated annealing Gauss disturbance bat algorithm, the idea of simulated annealing algorithm into the BA algorithm, and then use the BA algorithm to find the optimal solutions, and the current position of the Gauss perturbation, the algorithm has achieved good results ${ }^{[10]}$.

\section{Bat algorithm}

The bat algorithm (BA) is a new heuristic algorithm ${ }^{[6]}$, it is proposed by XinShe Yang in 2010. Yang illustrates the basic idea of this algorithm and puts forward the basic assumption of the bat algorithm:

1) All bats use their echolocation perception and the distance of the target, they identify the target and the different of the background obstacles in a mysterious way.

2) The location of bats are $x_{i}$, flying with the speed of $v_{i}$, they search target at a fixed frequency $f$, variable wavelength $\lambda$ and loudness $A_{i}$. They can determine the distance between himself and the prey and adjust the pulse wavelength (frequency) automatically, at the same time, when they close to the target, they adjust the pulse frequency $f_{i} \in[0,1]$. 
3) There are a lot of changes in loudness, the assumption it is from the maximum value of $A_{\max }$ changes to the fixed minimum value of $A_{\min }$.

\section{A bat algorithm by using maneuvering flight (MFBA)}

In flight, bats have two species of: one is the normal non motorized flight when there is no obstacle or natural enemy. One is the flexible and changeable maneuver when faced with obstacles or natural enemies. Secondly, the position of, the speed of flight and the direction of the flight of the bat in the T, and the position of, the speed of flight and the distance between the target in the T -1 .

In this paper, the flight activity of bats is as follows: 1.The two kinds of flight modes of non maneuvering flight and maneuver flight are assumed. 2. The bat has a strong flying skills, and only according to their own current position and the current optimal position to determine their next mobile position. This paper proposed a bat algorithm by using maneuver flight (MFBA). Finally, the simulation results show that the MFBA algorithm has better search ability, and the overall performance of the bat algorithm is improved.

MFBA algorithm design is as follows: the position of bat $i$ at $\mathrm{t}-1$ is $X_{i}^{t-1}=\left(X_{i 1}^{t-1}, \ldots, X_{i d}^{t-1}\right)$ , the group find the best position is $X_{*}=\left(x_{* 1}, \ldots, x_{* d}\right)$.

1. If the flight of the bat $i$ as follows:

$$
X_{\mathrm{i}}^{\mathrm{t}}=X_{i}^{t-1}+S\left(\frac{X_{i}^{t-1}-X_{*}}{\left\|X_{i}^{t-1}-X_{*}\right\|}\right)
$$

Where $\mathrm{s}$ is $(0,1)$ uniformly random distribution. The bat $i$ is the non motorized flight at $t$.

2. If the flight of the bat $i$ as follows: $X_{\mathrm{ij}}^{\mathrm{t}}=X_{* k}^{t-1}, j=1,2,3 \ldots, d$.

Where $k$ is random number in the set of $\{1, \ldots, d\}$. The $j$ dimension or bit of the bat $i$ around the $k$ dimension of $X_{*}=\left(\mathrm{x}_{* 1}, \ldots, x_{* d}\right)$, it is said that the bat $i$ using a motorized flight mode at $\mathrm{t}$.

The bat $i$ according to the pros and cons of the current position, according to the following rules to determine its flight mode.

$$
\left\{\begin{array}{l}
X_{\mathrm{ij}}^{\mathrm{t}}=X_{* k}^{t-1} \quad \text { if } \delta \geq s_{i}(t) / n \\
X_{i}^{t}=X_{i}^{t-1}+S\left(\frac{X_{i}^{t-1}-X_{*}}{\left\|X_{i}^{t-1}-X_{*}\right\|}\right)
\end{array}\right.
$$

Where $\delta$ is the uniform random distribution in $(0,1)$.

\section{The Algorithm flow}

The basic flow of MFBA algorithm is as follows:

Step1 Initialization the position of $X_{i}$, step parameters, pulse emission rate of $\gamma$ and loudness $A_{i}$ of the bat $i$, and, the optimal initial position of group $n$ bat as the initial $X$ *

Step2 The bat $i$ adjust its flight mode according to formula 2 to update location.

Step3 The fitness function $f\left(X_{i}\right)$ of the bat $i$.

Step4 If randomly generated a random number of rand is greater than pulse rate $\gamma$ of the bat $i$, the bat $i$ according to the formula $X_{i}^{\mathrm{t}+1}=X_{\mathrm{i}}^{\mathrm{t}}+\alpha \oplus \operatorname{Levy}(\lambda)$ to generate a local solution near the optimal solution $X_{\text {new }}$.

Step5 If randomly generated a random number of rand is less than loudness $A_{i}$ the bat $i$, the bat $i$ according to the formula $A_{\mathrm{i}}^{\mathrm{t}+1}=\alpha A_{\mathrm{i}}^{\mathrm{t}}$ to generate a local solution near the optimal solution $X_{\text {new }}$.

Step6 According to the fitness value of the size of the bat to sort, find the current optimal position $X_{*}$. 
Step7 Once the end of the iteration 1, determine whether to reach the stop conditions, if achieve the stop conditions, then the end of the algorithm and the output of the optimal location of the bat and its fitness function, otherwise the next iteration to go to Step2.

\section{The analysis of simulation}

In order to verify the performance of the MFBA algorithm, we use $\mathrm{C}++$ to develop a human motion tracking system, this experiment uses the computer for AMD Athlon (tm) IIX2B24, 2GB memory PC, programming software for the Matlab2010a. Experiments of the sports video use a high degree of error in parallel optical axis camera. Figure 1 is the result of a sequence of images taken from a sequence of images, Figure 2 Human body motion 3D skeleton positive views, Figure 2 Human body motion 3D skeleton side views.

In order to the comparable, in the performance test of the algorithm, the parameters of the MFBA algorithm and the BA algorithm are set to be consistent. Specific parameters are set as follows: group size $n=40 \quad \alpha=\beta=0.9, f_{\max }=100, f_{\min }=0$; the maximum number of iterations is 200 times.

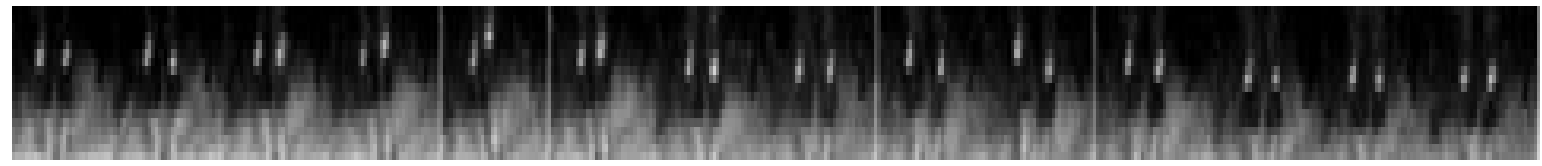

Figure 1 The result of a sequence of images taken from a sequence of images

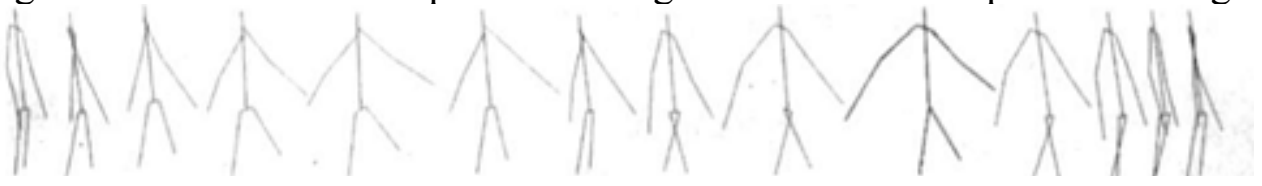

Figure 2 Human body motion 3D skeleton positive views
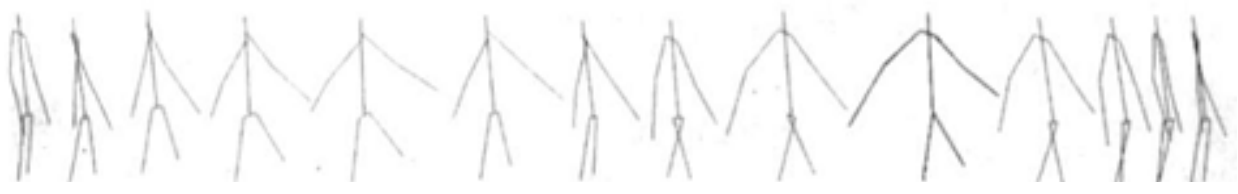

Figure 3 Human body motion 3D skeleton side views

\section{Conclusions}

In this paper, a new algorithm of MFBA is proposed, the algorithm is based on the maneuver flight. Each bat chooses different flight patterns according to the position of the bat in the algorithm. Experimental results show that MFBA has a fast convergence speed and good accuracy, which can avoid falling into local optimal solution and has strong global search ability.

\section{References}

[1] Yang X S. A new metaheuristic bat-inspired algorithm[J]. Nature Inspired Cooperative Strategies for Optimization (NICSO 2010) (Eds. J. R. Gonzalez et al.), Springer, 2010, 284: 65-74.

[2] Yang X S, Bat Algorithm for Multi-objective Optimization[J]. International Journal of Bio-Inspired Computation Bio-Inspired Computation (IJBIC.) 2011, 3(5):267-274.

[3] Tsai P W, Pan J S, Liao B Y, et al. Bat Algorithm Inspired Algorithm for Solving Numerical Optimization Problems[J], Applied Mechanics and Materials, 2011,148:134-137.

[4] Khan K, Nikov A, Sahai A. A Fuzzy Bat Clustering Method for Ergonomic Screening of Office Workplaces, S3T 2011[J]. Advances in Intelligent and Soft Computing, 2011, 101:59-66.

[5] Tamiru A L, and Fakhruldin M H. Application of Bat Algorithm and Fuzzy Systems to Model Exergy Changes in a Gas Turbine Artificial Intelligence[J]. Evolutionary Computation and Metaheuristics (AIECM), 2011, 112: 437-442. 
[6] Mishra S, Shaw K, Mishra D. A new metaheuristic classification approach for microarray data[J].Procedia Technology,2012 4(1):802-806.

[7] Bora T C, Coelho L S, Lebensztajn L. Bat-Inspired Optimization Approach for The Brushless DC Wheel Motor Problem, IEEE Transactions On Magnetics, 2012, 48(2): 947-950.

[8] Li Zhiyong, Ma Liang, Zhang Huizhen. Genetic mutation bat algorithm for 0-1 knapsack problem[J]. computer engineering and applications, 2014, 50(11):49-52.

[9] Huang Guangqiu, Zhao Weijuan, Lu Qiuqin. Bat algorithm with global convergence for solving large-scale optimization problem [J]. Application Research of computers, 2013,30(5): 1323-1328.

[10] He Xingshi, Ding Wenjing, Yang Xin. Bat algorithm based on simulated annealing and Gaussian perturbations [J]. Application Research of computers, 2014, 31 (2): 392-397. 